\title{
The Effect of Regret-Based Risky Route Choice on the Traffic Equilibrium for Emergency Evacuation
}

\author{
Ze Wang $D^{1,2}$ Haiqiang Yang, ${ }^{3}$ and Linglin $\mathrm{Ni}^{4}$ \\ ${ }^{1}$ Institute of Intelligent Transportation Systems, Zhejiang University, Hangzhou 310058, China \\ ${ }^{2}$ Department of Traffic Management Engineering, Zhejiang Police College, Hangzhou 310053, China \\ ${ }^{3}$ Institute for Future (IFF), Qingdao University, Qingdao 266071, China \\ ${ }^{4}$ Department of Business Management, Zhejiang University of Finance and Economics Dongfang College, \\ Hangzhou 310012, China
}

Correspondence should be addressed to Ze Wang; wangze2@zjjcxy.cn

Received 26 May 2020; Revised 13 September 2020; Accepted 27 September 2020; Published 12 October 2020

Academic Editor: Yajie Zou

Copyright (c) $2020 \mathrm{Ze}$ Wang et al. This is an open access article distributed under the Creative Commons Attribution License, which permits unrestricted use, distribution, and reproduction in any medium, provided the original work is properly cited.

Following the research on human decision-making under risk and uncertainty, the purpose of this paper is to analyze evacuees' risky route decision behavior and its effect on traffic equilibrium. It examines the possibility of applying regret theory to model travellers' regret-taking behavior and network equilibrium in emergency context. By means of modifying the utility function in expected utility theory, a regret-based evacuation traffic equilibrium model is established, accounting for the evacuee's psychological behavior of regret aversion and risk aversion. Facing two parallel evacuation routes choice situation, the effect of evacuees' risk aversion and regret aversion on traffic equilibrium is numerically investigated as well as the road capacity reduction from natural disaster. The findings reveal that evacuees prefer the riskless route with the lower travel time as the increase of the regret aversion degree. The equilibrium tends to be achieved when more evacuees choose the safer route jointly affected by risk aversion and regret aversion. Moreover, an optimization model for disaster occurring possibility is formulated to assess the traffic system performance for evacuation management. These findings are helpful for understanding how the regret aversion and risk aversion influence traffic equilibrium.

\section{Introduction}

Traffic equilibrium is a key process of transportation demand analysis and planning. The assumption of the presence of traffic equilibrium can help to predict route flow patterns in the network and evaluate the associated measures of system performance. It is critical to efficiently identify the potential periodic patterns from massive time-series data and provide accurate predictions for travel time and freeway traffic speed based on statistical, Markov chains and machine learning prediction models, especially for real evacuation scenario [1-3]. Previous studies have shown expected utility theory's popularity on depicting travel behaviors and solving traffic equilibrium problem [4-8]. It postulates that each individual traveler attempts to maximize the utility of the chosen route as the principle rule.
Travel time is an important indicator for traveller decision-making and traffic assignment. Besides travel time, other route spatiotemporal factors including intersection delay, path distance, and path size are also considered in the route choice process [9]. Current research on route choice turns to model travellers' responses to uncertainty. However, the principle assumption of expected utility maximization lacks behavioral realism in some risky decision-making occasions, especially the emergency evacuation context [10-12].

It is widely acknowledged that the notion of regret is highly relating to individual's decision-making [13]. The regret theory relaxes the traditional behavioral assumption and provides an opportunity to account for the regret aversion psychology, especially when people face risky choice decision-making [14]. A recent effort in modelling 
individual's travel choices using the regret theory has been made, such as route choice, mode choice, and departure times $[15,16]$. A small but growing body of studies has been performed to analyze traffic equilibrium based on the regret theory. The regret theory has great potential in modelling traffic equilibrium compared with expected utility theory and prospect theory [16-18]. The outstanding advantage lies in its parsimonious form to explain the actual behaviors and being consistent with empirical studies. A regret-based stochastic user equilibrium model has been proposed in more general choice sets that can depict the traveller's route choice behaviors more flexibly [19]. Considering the travellers' way of thinking, a noncompensatory multiobjective framework has been developed, and conflicts among multiple objectives can be solved through the model [20]. Since the monetary cost was also an important factor when making a decision between alternative routes, a biattribute user equilibrium model (i.e., travel time and monetary cost) has been established in which travellers aim to minimise their regret [21].

In recent years, although the prospect theory is increasingly applied to explain traveler's risky route choice behaviors, it is difficult to select a scientific reference point [22-24]. A unique pure nash equilibrium point has been achieved under the principles of prospect theory considering users' diverse behavioral patterns [25]. Cloud computing environments, flexible 5G access technology, and clustering mechanisms may help enhance evacuees' mobility characteristics [26-28]. The regret aversion psychology was conformed to affect the traffic equilibrium in the studies conducted by Chorus [17]. The impacts of regret and risk aversions increase and appear to reinforce one another, both implying equilibrium shifts towards safer routes. Regret is weaker in the environment with weaker risk degree, but it is stronger in the risky environment [29].

The review of the existing literature reveals that few studies focus on the traffic equilibrium with regret aversion under evacuation risky decision-making process. Evacuees' decision behaviors when facing the emergency evacuation are different from that in the regular conditions; thus their regret and risk aversion should be considered [30, 31]. Therefore, the application of the regret-based model in the field of emergency transportation needs to be addressed urgently. One motivation of this study is to analyze the traffic equilibrium under evacuation condition based on the regret theory. Meanwhile, the global optimization model for disaster state is constructed to assess the performance of traffic system in order to make feasible evacuation guidance. The analysis of the traffic equilibrium is the premise of assessing the performance of traffic system. The contribution of this study highlights to capture unobserved risk and regret aversions psychology in the evacuation decision-making process.

The remainder of this paper is organized as follows. Section 2 presents the regret-based traffic equilibrium models for emergency evacuation. Section 3 explores how the risk and regret aversions affect the evacuation traffic equilibrium, and Section 4 formulates the system performance assessment model. The Section 5 concludes the study and discusses future research directions.

\section{Regret-Based Traffic Equilibrium Models}

Travel behavior has a potential significant impact on the traffic equilibrium. In contrast to previous studies based on utility framework, a regret-based traffic equilibrium model was formulated accounting for risk and regret aversion decisions. The psychological regret/rejoice may occur when the alternative is worse/better than other alternatives. The decision-makers try to avoid the higher regret, specifically regret aversion. Facing a risky decision-making situation, more attentions were paid on regret theory rather than traditional utility theory.

2.1. Regret-Based Utility Functions. Assume a situation that evacuees should leave home for a safe destination to avoid the damage from natural disaster (e.g., flood and storms) through main evacuation routes $a$ or $b$ with risky travel time as shown in Figure 1. The route $a$ is closer to the disaster than route $b$, but the route distance is shorter. The perceived travel time is uncertain, and its distribution is known to evacuees. More specifically, the evacuee knows the occurrence probability of random travel time $p_{r}$ for every disaster state. Three different states $s$ of the disaster including "good state," "medium state," and "bad state" may occur with the occurrence probability $p_{s}$.

According to the regret theory, the perceived utility function consists of two parts: basic utility function and regret-rejoice function $[10,12]$. The regret-based utilities for the two routes are shown as follows:

$$
R U(a)=U\left(t_{s a}\right)+R[\Delta U(a)]=U\left(t_{s a}\right)+R\left[U\left(t_{s a}\right)-U\left(t_{s b}\right)\right],
$$

$R U(b)=U\left(t_{s b}\right)+R[\Delta U(b)]=U\left(t_{s b}\right)+R\left[U\left(t_{s b}\right)-U\left(t_{s a}\right)\right]$,

where $U\left(t_{s a}\right)$ and $U\left(t_{s b}\right)$ represent the basic utility of route $a$ and $b ; t_{s a}$ and $t_{s b}$ are the travel time of routes $a$ and $b$ in the disaster state $s$; and $R[\Delta U(a)]$ and $R[\Delta U(b)]$ represent the regret-rejoice value of route $a$ and $b$. When $R[\Delta U(a)]>0$, it becomes a rejoice value, indicating that the evacuee perceives the rejoice psychology from choosing route $a$. When $R[\Delta U(b)]<0$, it becomes a regret value, indicating that the evacuee perceives the regret psychology from choosing route $b$. Notably, the part $R(\Delta U)$ is a function of the utility difference between route $a$ and $b$.

There are different forms depending on the risk aversion level for the utility function $U$. For $a$ cost-attribute (e.g., travel time), $U$ is a monotonically decreasing concave function, and it satisfies the conditions $U^{\prime}<0$ and $U^{\prime \prime}(\Delta U)>0[32,33]$. For a benefit-attribute (e.g., service), $U$ is a monotonically increasing concave function, and it satisfies the conditions $U^{\prime}>0$ and $U^{\prime \prime}(\Delta U)<0$. The exponential expression was applied as the utility function for travel time attribute as follows $[10,12,17]$ :

$$
U\left(t_{s}\right)=\frac{1-\exp \left(\alpha \cdot t_{s}\right)}{\alpha},
$$




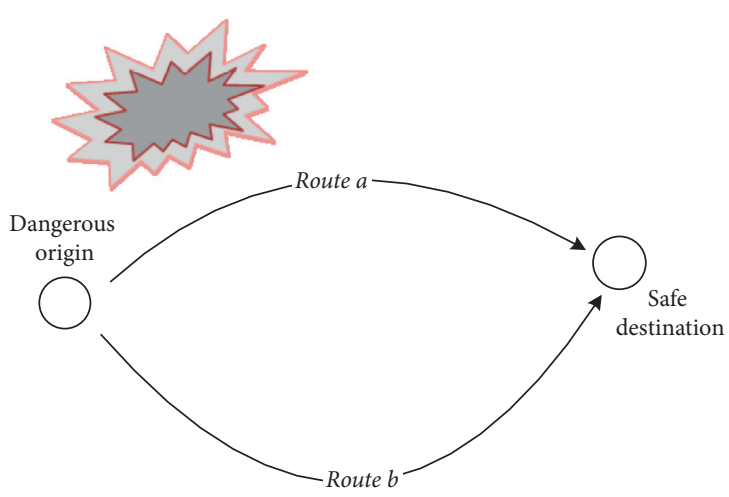

Figure 1: A schematic description of the route choice decision.

where $\alpha$ represents a nonnegative risk aversion parameter and it ranges from 0 to 1 . Generally, the decision-makers are risk averse when facing risky choices. The influence of risk aversion on the utility with respect to travel times is shown in Figure 2. It is found that the risk aversion level of the evacuee is increasing with risk aversion parameter.

The regret-rejoice function is highly dependent on the regret-aversion level. In most situations, the evacuees are risk aversion when facing disaster. The function $R(\Delta U)$ is a monotonically increasing concave function, and it satisfies the conditions $R^{\prime}(\Delta U)>0, \quad R^{\prime \prime}(\Delta U)<0$ and $R(0)=0$ $[33,34]$. The regret-rejoice utility with regret aversion parameter is shown as follows $[10,12,17]$ :

$$
R(\Delta U)=1-\exp (-\beta \cdot \Delta U)
$$

where $\beta$ represents a nonnegative regret aversion parameter, and it ranges from 0 to 1 . The influence of regret aversion on the utility difference is shown in Figure 3. When $\Delta U>0$, $|R(-\Delta U)|>R(\Delta U)$ is found with increase in $\beta$. It reveals that the perceived psychology to $-\Delta U$ is more sensitive than that to $\Delta U$ for an evacuee. In other words, the evacuees are regret averse, and the regret aversion level increases with the increases in the risk aversion parameter.

Consequently, considering the risky travel time, the regret-based utility for route $a$ and $b$ can be written as follows:

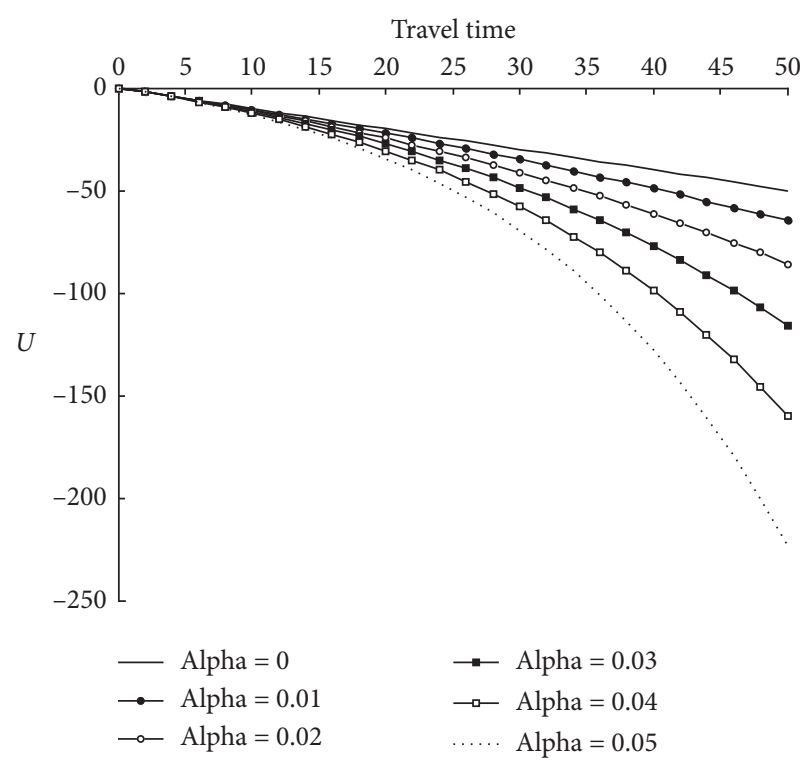

FIGURE 2: Influence of risk aversion on the utility with respect to travel times.

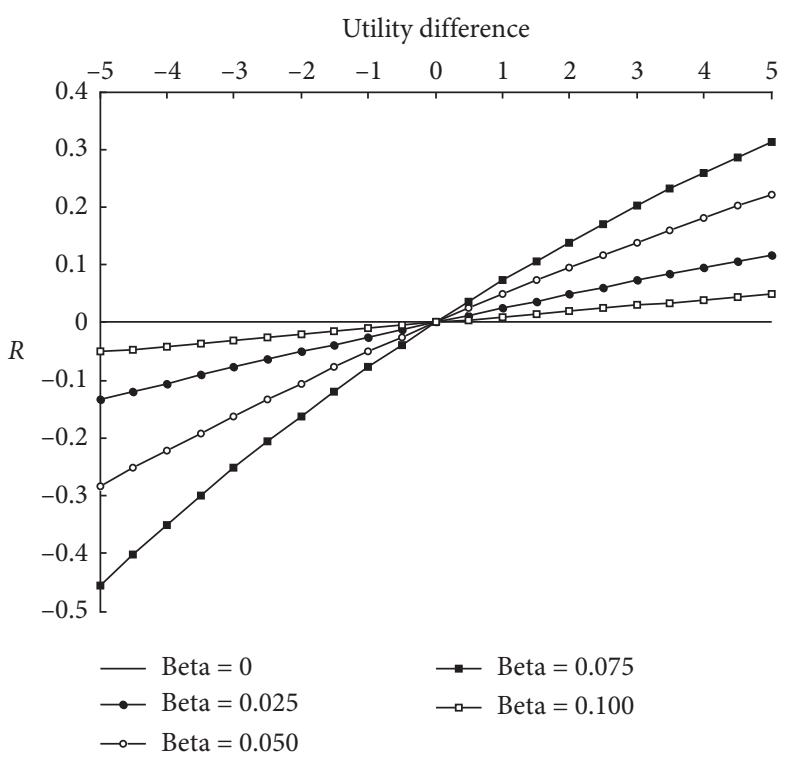

Figure 3: Influence of regret aversion on the utility difference.

$$
\begin{aligned}
& \operatorname{ERU}(a)=\sum_{s} \sum_{r} p_{s} \cdot p_{r} \cdot\left(\left[\frac{1-\exp \left(\alpha \cdot t_{s a}\right)}{\alpha}\right]-\left[1-\exp \left(-\beta \cdot\left[\frac{\exp \left(\alpha \cdot t_{s b}\right)-\exp \left(\alpha \cdot t_{s a}\right)}{\alpha}\right]\right)\right]\right) \\
& E R U(b)=\sum_{s} \sum_{r} p_{s} \cdot p_{r} \cdot\left(\left[\frac{1-\exp \left(\alpha \cdot t_{s b}\right)}{\alpha}\right]-\left[1-\exp \left(-\beta \cdot\left[\frac{\exp \left(\alpha \cdot t_{s a}\right)-\exp \left(\alpha \cdot t_{s b}\right)}{\alpha}\right]\right)\right]\right)
\end{aligned}
$$

2.2. Traffic Equilibrium Conditions. From the perspective of behavior science, individual route choice decisions lead to the transportation network flow pattern evolving to traffic equilibrium [29]. More specifically, no user can decrease his utility by unilaterally switching routes. In this study, traffic equilibrium condition can be extended into a regret-based decision framework. The equilibrium is achieved when no user can increase his regret-based utility by unilaterally switching routes. According to user equilibrium principle proposed by Wardrop [35], the network is considered in equilibrium state when all traffic patterns stabilize and no user has any incentive to change its current route, as shown as follows: 


$$
\begin{cases}f(a)=0, & \text { if } \operatorname{ERU}(a)<\operatorname{ERU}(b), \\ f(b)=0, & \text { if } \operatorname{ERU}(a)>\operatorname{ERU}(b), \\ f(a) \cdot f(b) \neq 0, & \text { if } \operatorname{ERU}(a)=\operatorname{ERU}(b) .\end{cases}
$$

The disaster has a destructive effect on the road capacity. In order to capture this influence on network equilibrium in the real evacuation context, a capacity reduction parameter is introduced to BPR (Bureau of Public Road, BPR) function form adopted in Avineri [36] as follows:

$$
t_{s a}=t_{s a f} \cdot\left[1+k \cdot\left(\frac{Q_{s a}}{\gamma_{s a} \cdot C_{s a}}\right)^{\lambda}\right]+t_{s a r},
$$

where $t_{\text {saf }}$ is free-flow travel time for route $a$ under the state $s ; k$ and $\lambda$ are the parameters; $Q_{s a}$ is the traffic flow on the route $a$ under the state $s$; $C_{s a}$ is the basic capacity of the route $a$ under the state $s ; \gamma_{s a}$ is the road capacity reduction parameter under the state $s$; the smaller the $\gamma$ value, the greater the capacity loss; and $t_{\text {sar }}$ is the random travel time of the route $a$ under the state $s$.

Here, the BPR function is used as a route travel cost function when the links on one route are homogeneous (i.e., the capacity and the service level are the same) like Avineri [36]. Substituting equation (8) in equations (5) and (6), the network travel flow and user utility can be obtained in traffic equilibrium state.

\section{Risk and Regret Aversion Parameter Analysis}

One numerical example is conducted in this study to recognize the different effects of risk aversion level and regret aversion level on traffic equilibrium. The values of assumed parameters are partly taken from Chorus [17]. In contrast to the study done by Chorus, the regret-based traffic equilibrium is analyzed under emergency evacuation context, considering the effect of disaster on road capacity. Route $a$ is closer to the disaster than route $b$ but the route distance is shorter in Figure 1, it means route $a$ is more likely to be affected by the disaster and suffer a greater capacity reduction in the emergency situation. For detailed information on the numerical example, the following settings are assumed: $t_{s a f}=10, t_{s b f}=12, k=\lambda=2$, and $C_{a}=C_{b}=200$. There are 200 people that should be evacuated. With regards to the three states, the random parts of travel time and the capacity reduction parameters are different due to the damage degree of the disaster, as shown in Table 1. For the three states, the occurrence probability of the random parts of travel time is assumed to $1 / 3$.

3.1. Different Risk Aversion Scenarios. Take the good state as the example, the parameter settings are assumed: $\beta=0.05$, $\gamma_{a}=0.85$, and $\gamma_{b}=1$. When $Q_{a}$ denotes the traffic flows on the route $a$, the expected regret-based utility of route $a$ is captured when $\alpha$ varies from 0 to 0.05 , as shown in Figure 4 . It is found that the expected regret-based utility of route $a$ decreases with the increase in the risk aversion parameter.

When $\beta=0.05, \gamma_{a}=0.85$, and $\gamma_{b}=1$, the traffic flow achieves different equilibrium when $\alpha$ varies from 0 to 0.05 , as shown in Figure 5. The traffic flows on the route $a$ at the traffic equilibrium state are $104,101,99,96,92$, and 88 when $\alpha$ equals to $0,0.01,0.02,0.03,0.04$, and 0.05 , respectively. Take the case of $\alpha=0.02$ as an example, the traffic flow on the route $a$ is 99 cars, and the expected travel time is 31.8 , compared with 101 cars on the route $b$ and expected travel time of 33.1. As a result, the travel flow shifts from route $a$ towards route $b$, when the risk aversion parameter increases.

3.2. Different Regret Aversion Scenarios. Take the good state as the example, the parameter settings are assumed: $\alpha=0.03$, $\gamma_{a}=0.85$, and $\gamma_{b}=1$. When $Q_{a}$ denotes the traffic flows on the route $a$, the expected regret-based utility of route $a$ is captured when $\beta$ varies from 0 to 0.1 , as shown in Figure 6 . It is found that the expected regret-based utility of route $a$ decreases as the increase of regret aversion parameter.

When $\alpha=0.03, \gamma_{a}=0.85$, and $\gamma_{b}=1$, the traffic flow achieves different equilibrium when $\beta$ varies from 0 to 0.1 , as shown in Figure 7. The traffic flows on the route $a$ at the traffic equilibrium state are $96,96,95,95$, and 94 when $\beta$ equals to $0,0.025,0.050,0.075$, and 0.100 , respectively. Take the case of $\beta=0.075$ as an example, the traffic flow on the route $a$ is 95 cars, and the expected travel time is 31.2, compared with105 cars on the route $b$ and expected travel time of 33.6. As a result, the travel flow shifts from route $a$ towards route $b$, when the risk aversion parameter increases.

3.3. Different Risk-Regret Aversion Scenarios. The traffic equilibrium without risk and regret aversions is shown in Figure 8. It can be seen that there are 104 cars on the route $a$ and 96 cars on the route $b$ at traffic equilibrium state. Expected travel times on the two routes equal 32.5. These findings are certain in line with the study conducted by Chorus [16]. The value 104 is smaller than 114 provided by Chorus; it is due to the fact that the capacity reduction is considered in this study.

The traffic equilibrium with different risk and regret aversions in each state is shown in Table 2. It can be seen that the traffic flows on the route $a$ at the equilibrium state are the same when there is no risk aversion (i.e., $\alpha=0$ ) whatever $\beta$ varies. The traffic flows on route $a$ are 104, 97, and 91 in the good, medium, and bad states, respectively. As shown in Table 2, the traffic flow on the route $a$ shifts seriously with the regret aversion parameter increase when the risk aversion parameter increases simultaneously. This finding is consistent with that of the work done by Chorus [16]. In other words, the influence of increase in regret aversion on traffic equilibrium is larger for the traveler with more risk aversion. The impacts of increases in regret aversion and risk aversion appear to reinforce one another, both implying traffic equilibrium shifts towards safer routes (i.e., route $b$ ).

With respect to the capacity reduction parameter, the disaster damage degree reinforces the impacts from the regret aversion and risk aversion further. The traffic equilibrium for the route $a$ is 101 when $\alpha=0.04$ without damage. However, the traffic equilibrium is 93 when $\alpha=$ 0.04 with $a$ capacity reduction on route a (i.e., $\gamma_{a}=0.85$ ), indicating that evacuees are more alike to choose the less damage routes. 
TABLE 1: Random travel time and capacity reduction parameters for the three states.

\begin{tabular}{|c|c|c|c|c|}
\hline Route & Parameters & Good state & Medium state & Bad state \\
\hline \multirow{4}{*}{ Route $a$} & & 0 & 5 & 10 \\
\hline & Random travel time & 15 & 20 & 25 \\
\hline & & 30 & 35 & 40 \\
\hline & Capacity reduction parameter & 0.85 & 0.75 & 0.65 \\
\hline \multirow{4}{*}{ Route $b$} & & 12.5 & 15 & 17.5 \\
\hline & Random travel time & 15 & 17.5 & 20 \\
\hline & & 17.5 & 20 & 22.5 \\
\hline & Capacity reduction parameter & 1.00 & 0.85 & 0.75 \\
\hline
\end{tabular}

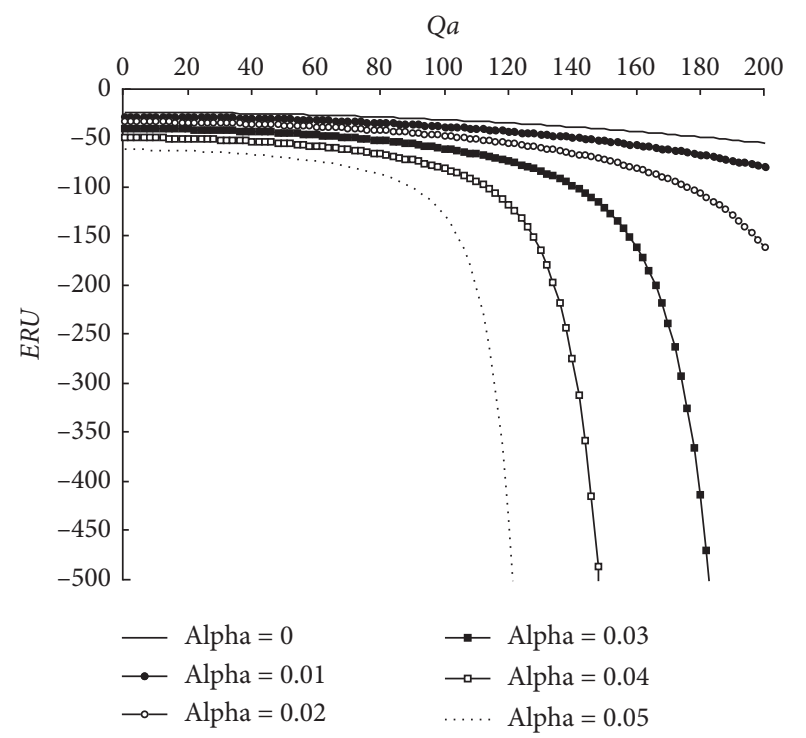

FIGURE 4: Expected regret-based utility of route a based on different risk aversion scenarios.

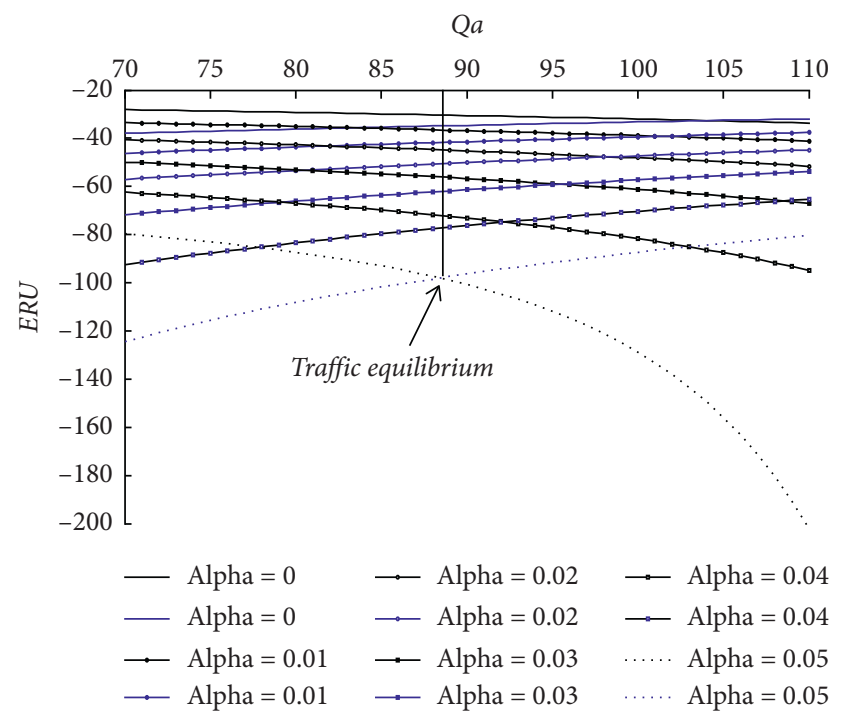

FIGURE 5: Influence of risk aversion parameter on traffic equilibrium. Note: the black line denotes route $a$, and the blue line denotes route $b$.

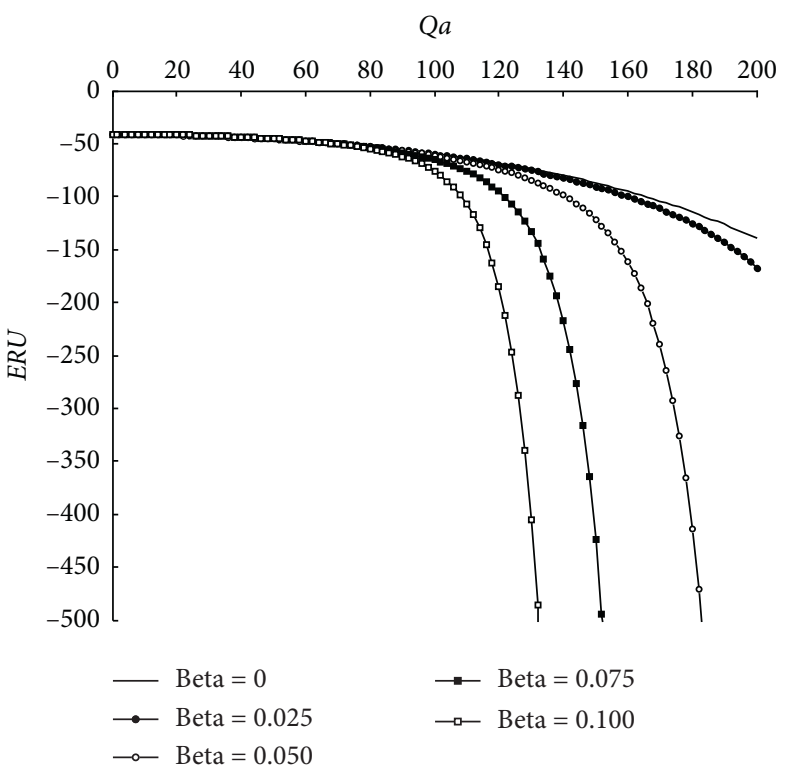

FIGURE 6: Expected regret-based utility of route $a$ based on different regret aversion scenarios.

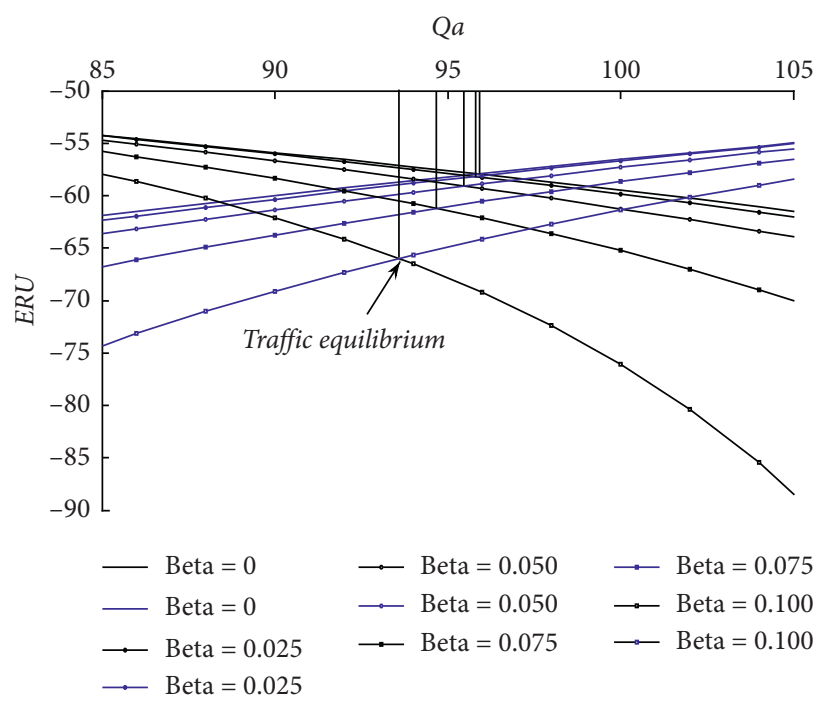

FIGURE 7: Influence of regret aversion parameters on traffic equilibrium. Note: the black line denotes route $a$, and the blue line denotes route $b ; Q_{b}=200-Q_{a}$. 


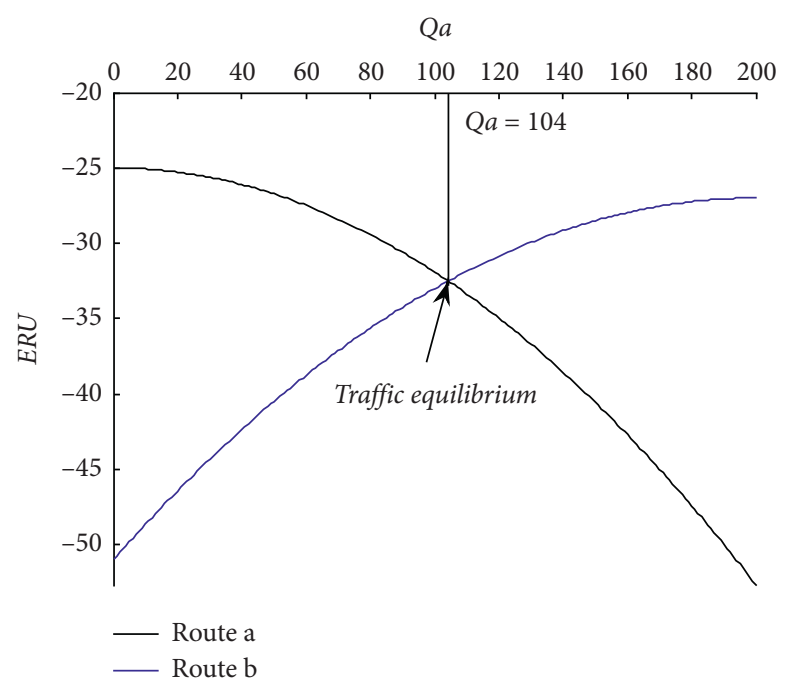

FIGURE 8: Expected regret-based utility without risk and regret aversion. Note. the black line denotes route $a$, and the blue line denotes route $b ; Q_{b}=200-Q_{a}$.

TABLE 2: Influence of risk aversion and regret aversion on traffic equilibrium.

\begin{tabular}{|c|c|c|c|c|c|c|c|}
\hline$\beta$ & $\gamma_{a}$ and $\gamma_{b}$ & $\alpha=0$ & $\alpha=0.01$ & $\alpha=0.02$ & $\alpha=0.03$ & $\alpha=0.04$ & $\alpha=0.05$ \\
\hline \multirow{4}{*}{$\beta=0$} & $\gamma_{a}=\gamma_{b}=1.00$ & 114 & 110 & 107 & 104 & 101 & 98 \\
\hline & $\gamma_{a}=0.85, \gamma_{b}=1.00$ & 104 & 101 & 99 & 96 & 93 & 91 \\
\hline & $\gamma_{a}=0.75, \gamma_{b}=0.85$ & 97 & 95 & 93 & 91 & 89 & 87 \\
\hline & $\gamma_{a}=0.65, \gamma_{b}=0.75$ & 91 & 89 & 88 & 86 & 84 & 83 \\
\hline \multirow{4}{*}{$\beta=0.025$} & $\gamma_{a}=\gamma_{b}=1.00$ & 114 & 110 & 107 & 104 & 101 & 98 \\
\hline & $\gamma_{a}=0.85, \gamma_{b}=1.00$ & 104 & 101 & 99 & 96 & 93 & 90 \\
\hline & $\gamma_{a}=0.75, \gamma_{b}=0.85$ & 97 & 95 & 93 & 91 & 89 & 86 \\
\hline & $\gamma_{a}=0.65, \gamma_{b}=0.75$ & 91 & 89 & 88 & 86 & 84 & 82 \\
\hline \multirow{4}{*}{$\beta=0.05$} & $\gamma_{a}=\gamma_{b}=1.00$ & 114 & 110 & 107 & 104 & 100 & 95 \\
\hline & $\gamma_{a}=0.85, \gamma_{b}=1.00$ & 104 & 101 & 99 & 96 & 92 & 88 \\
\hline & $\gamma_{a}=0.75, \gamma_{b}=0.85$ & 97 & 95 & 93 & 90 & 87 & 84 \\
\hline & $\gamma_{a}=0.65, \gamma_{b}=0.75$ & 91 & 89 & 87 & 85 & 83 & 80 \\
\hline \multirow{4}{*}{$\beta=0.075$} & $\gamma_{a}=\gamma_{b}=1.00$ & 114 & 110 & 107 & 103 & 98 & 93 \\
\hline & $\gamma_{a}=0.85, \gamma_{b}=1.00$ & 104 & 101 & 98 & 95 & 91 & 86 \\
\hline & $\gamma_{a}=0.75, \gamma_{b}=0.85$ & 97 & 95 & 92 & 89 & 86 & 83 \\
\hline & $\gamma_{a}=0.65, \gamma_{b}=0.75$ & 91 & 89 & 87 & 85 & 82 & 80 \\
\hline \multirow{4}{*}{$\beta=0.100$} & $\gamma_{a}=\gamma_{b}=1.00$ & 114 & 110 & 106 & 101 & 96 & 91 \\
\hline & $\gamma_{a}=0.85, \gamma_{b}=1.00$ & 104 & 101 & 98 & 94 & 89 & 85 \\
\hline & $\gamma_{a}=0.75, \gamma_{b}=0.85$ & 97 & 94 & 92 & 88 & 85 & 82 \\
\hline & $\gamma_{a}=0.65, \gamma_{b}=0.75$ & 91 & 88 & 87 & 84 & 82 & 80 \\
\hline
\end{tabular}

\section{Traffic System Performance Assessment}

From the viewpoint of decision-making department, it is helpful to know the global traffic system performance. Because the occurrence probability of the disaster at different states is uncertain, the traffic system performance is not a determined value. The global utility of traffic system at equilibrium can be considered as a performance indicator. To recognize this point, the interval value is used to present the occurrence probability. In detail, the interval values for occurrence probabilities of the good state, medium state, and bad state are assumed to be [0.2,
$0.5],[0.4,0.7]$, and $[0.1,0.3]$, respectively. The best state and the worst state of the traffic system can be estimated using the global optimal state model. For every disaster state, based on the perceived regret-based utility of the routes, the global utility of the traffic system using an optimization model as follows:

$$
f(s)=\sum_{s} \sum_{r} p_{s} \cdot E R U_{s r}
$$

In this study, the utilities for the three states when $\alpha=$ 0.04 and $\beta=0.1$ are used. The global optimal occurrence probability can be obtained using the following model: 


$$
\begin{gathered}
f(s)=-161.12 p_{1}-169.28 p_{2}-240.84 p_{3}, \\
\left\{\begin{array}{c}
0.2 \leq p_{1} \leq 0.5 \\
0.4 \leq p_{2} \leq 0.7 \\
0.1 \leq p_{3} \leq 0.3 \\
p_{1}+p_{2}+p_{3}=1 .
\end{array}\right.
\end{gathered}
$$

At last, the global optimal state probabilities can be obtained through solving equation (10). The best utility of traffic system at the network equilibrium is -172.356 that mainly depends on the good state and medium state, that is, $p_{1}=0.5, p_{2}=0.4$, and $p_{3}=0.1$. The increase of global utility due to the action on bad state is relatively limited. The worst utility of traffic system at the network equilibrium is -189.116 that mainly depends on the bad state and medium state, that is, $p_{1}=0.2, p_{2}=0.5$, and $p_{3}=0.3$. The interval value of traffic system utility is between -189.116 and -172.356 . The performance of traffic system can obtain a minimum regret-based disutility at 172.356 . According to the model results, the findings provide useful insights for the emergency agency that the management department should pay more attentions on the slight and medium disaster damage.

\section{Conclusions}

A regret-based utility function was used to account for the risk aversion and regret aversion psychology under emergency context. Meanwhile, the traffic equilibrium conditions were constructed considering the road capacity reduction. It is found that risk aversion, regret aversion, and capacity reduction have important effects on traffic equilibrium.

How risk aversion and regret aversion parameters influence the traffic equilibrium was explored. It is found that the traffic equilibrium tends to be achieved when safer routes are the main choice. It reflects that most evacuees are willing to choose the safer routes in the evacuation text. They are highly risk and regret aversion, and the outcome of the traffic equilibrium is dependent on the risk aversion and regret aversion levels.

The traffic equilibrium model under regret theory is an appealing approach compared with traditional utility theory, especially in the evacuation traffic context. It is reasonable that aversion psychology is taken into the analysis of evacuation issues. Different parameters produce different degree of effects on the travel decision. With the increase of aversion levels, evacuees prefer the stable routes. Future research can be found in a few directions. The application of the regret-based traffic equilibrium model can be conducted in complicated network. For example, in a network with three evacuation routes $a, b$, and $c, R U(a)$ can be modified to equal $U\left(t_{s a}\right)+R\left[U\left(t_{s a}\right)-\max \left\{U\left(t_{s a}\right), U\left(t_{s b}\right), U\left(t_{s c}\right)\right\}\right]$. Meanwhile, facing a risk situation, evacuees' psychological behavior of regret aversion should not be all the same. The heterogeneity of regret-taking behavior should be taken into consideration in the future study. Real field data also should be utilized to validate the conclusion, and implementation cost can be well evaluated for evacuation practice.

\section{Data Availability}

The data used to support the findings of this study are available from the corresponding author upon request.

\section{Conflicts of Interest}

The authors declare that they have no conflicts of interest.

\section{Acknowledgments}

This work was mainly supported by the National Natural Science Foundation of China (no. 71704161), Zhejiang Provincial Natural Science Foundation of China (no. LGF19G030002), China Postdoctoral Science Foundation (no. 2017M621935), National Natural Science Foundation of China (no. 71801188), and Zhejiang Provincial Philosophical and Social Science Program (no. 19NDJC167YB).

\section{References}

[1] X. Yang, Y. Zou, J. Tang, J. Liang, and M. Ijaz, "Evaluation of short-term freeway speed prediction based on periodic analysis using statistical models and machine learning models," Journal of Advanced Transportation, vol. 2020, Article ID 9628957, 16 pages, 2020.

[2] J. Chen, K. Li, H. Rong, K. Bilal, K. Li, and P. S. Yu, “A periodicity-based parallel time series prediction algorithm in cloud computing environments," Information Sciences, vol. 496, pp. 506-537, 2019.

[3] J. Tang, J. Hu, W. Hao, X. Chen, and Y. Qi, "Markov chains based route travel time estimation considering link spatiotemporal correlation," Physica A: Statistical Mechanics and its Applications, vol. 545, 2020.

[4] T. A. Arentze and H. J. P. Timmermans, "Information gain, novelty seeking and travel: a model of dynamic activity-travel behavior under conditions of uncertainty," Transportation Research Part A, vol. 39, no. 2-3, pp. 125-145, 2005.

[5] A. de Palma and N. Picard, "Route choice decision under travel time uncertainty," Transportation Research Part A: Policy and Practice, vol. 39, no. 4, pp. 295-324, 2005.

[6] J. W. Polak, S. Hess, and X. Liu, "Characterizing heterogeneity in attitudes to risk in expected utility models of mode and departure time choice," in Proceedings of the 87th Annual Meeting of the Transportation Research Board, Washington, DC, USA, January 2008.

[7] R. D. Connors and A. Sumalee, "A network equilibrium model with travellers' perception of stochastic travel times," Transportation Research Part B: Methodological, vol. 43, no. 6, pp. 614-624, 2009.

[8] A. de Palma and N. Picard, "Equilibria and information provision in risky networks with risk-averse drivers," Transportation Science, vol. 40, no. 4, pp. 393-408, 2006.

[9] J. Tang, Y. Wang, W. Hao, F. Liu, H. Huang, and Y. Wang, “A mixed path size logit-based taxi customer-search model considering spatio-temporal factors in route choice," IEEE Transactions on Intelligent Transportation Systems, vol. 21, no. 4, pp. 1347-1358, 2020.

[10] D. E. Bell, "Regret in decision making under uncertainty," Operations Research, vol. 30, no. 5, pp. 961-981, 1982.

[11] C. G. Chorus, T. A. Arentze, and H. J. P. Timmermans, "A Random Regret-Minimization model of travel choice," 
Transportation Research Part B: Methodological, vol. 42, no. 1, pp. 1-18, 2008.

[12] G. Loomes and R. Sugden, "Regret theory: an alternative theory of rational choice under uncertainty," The Economic Journal, vol. 92, no. 368, pp. 805-824, 1982.

[13] T. Starver, "Anticipating regret: why fewer options may be better," Econometrica, vol. 76, no. 2, pp. 263-305, 2008.

[14] M. Boeri and L. Masiero, "Regret minimisation and utility maximisation in a freight transport context," Transportmetrica A: Transport Science, vol. 10, no. 6, pp. 548-560, 2014.

[15] C. G. Chorus, "A new model of random regret minimization," European Journal of Transport and Infrastructure Research, vol. 10, no. 2, pp. 181-196, 2010.

[16] C. Chorus, "Random regret minimization: an overview of model properties and empirical evidence," Transport Reviews, vol. 32, no. 1, pp. 75-92, 2012.

[17] C. G. Chorus, "Regret theory-based route choices and traffic equilibria," Transportmetrica, vol. 8, no. 4, pp. 291-305, 2012.

[18] G. M. Ramos, W. Daamen, and S. P. Hoogendoorn, "Expected utility theory, prospect theory, and regret theory compared for prediction of route choice behavior," Journal of Transportation Research Record, vol. 2230, no. 1, pp. 19-28, 2011.

[19] M. Li and H.-J. Huang, "A regret theory-based route choice model," Transportmetrica A: Transport Science, vol. 13, no. 3, pp. 250-272, 2017.

[20] S. Ye, S. Ma, and N. Jia, "A bi-attribute user equilibrium model considering travellers' regret aversion," Transportmetrica A: Transport Science, vol. 15, no. 2, pp. 1440-1458, 2019.

[21] Y. Yu, J. Zhou, and W. Xu, "Regret-based multi-objective route choice models and stochastic user equilibrium: a noncompensatory approach," Transportmetrica A: Transport Science, vol. 16, no. 3, pp. 473-500, 2020.

[22] S. Gao, E. Frejinger, and M. Ben-akiva, "Adaptive route choices in risky traffic networks: a prospect theory approach," Transportation Research Part C: Emerging Technologies, vol. 18, no. 5, pp. 727-740, 2010.

[23] Z. Li and D. Hensher, "Prospect theoretic contributions in understanding traveller behaviour: a review and some comments," Transport Reviews, vol. 31, no. 1, pp. 97-115, 2011.

[24] H. Xu, Y. Lou, Y. Yin, and J. Zhou, "A prospect-based user equilibrium model with endogenous reference points and its application in congestion pricing," Transportation Research Part B: Methodological, vol. 45, no. 2, pp. 311-328, 2011.

[25] P. Vamvakas, E. E. Tsiropoulou, and S. Papavassiliou, "Riskaware resource control with flexible $5 \mathrm{G}$ access technology interfaces," in Proceedings of the 2019 IEEE 20th International Symposium on "A World of Wireless, Mobile and Multimedia Networks" (WoWMoM), IEEE, Washington, DC, USA, June 2019.

[26] P. Vamvakas, E. E. Tsiropoulou, and S. Papavassiliou, “On the prospect of UAV-assisted communications paradigm in public safety networks," in Proceedings of the IEEE INFOCOM 2019-IEEE Conference on Computer Communications Workshops, IEEE, Paris, France, April 2019.

[27] E. E. Tsiropoulou, K. Koukas, and S. Papavassiliou, “A sociophysical and mobility-aware coalition formation mechanism in public safety networks," EAI Endorsed Transactions on Future Internet, vol. 18, no. 13, 2018.

[28] M. Shin, S. T. Shah, M. Y. Chung et al., "Moving small cells in public safety networks," in Proceedings of the 2017 International Conference on Information Networking (ICOIN), Da Nang, Vietnam, January 2017.
[29] P. Yuan and Z. Juan, "Risk route choice analysis and the equilibrium model under anticipated regret theory," PROMET-TrafficeTransportation, vol. 26, no. 1, pp. 33-43, 2014.

[30] C. G. Chorus, "Risk aversion, regret aversion and travel choice inertia: an experimental study," Transportation Planning and Technology, vol. 37, no. 4, pp. 321-332, 2014.

[31] P. Murray-tuite and B. Wolshon, "Evacuation transportation modeling: an overview of research, development, and practice," Transportation Research Part C: Emerging Technologies, vol. 27, pp. 25-45, 2013.

[32] C. E. Laciana and E. U. Weber, "Correcting expected utility for comparisons between alternative outcomes: a unified parameterization of regret and disappointment," Journal of Risk and Uncertainty, vol. 36, no. 1, pp. 1-17, 2008.

[33] H. Levy, "Absolute and relative risk aversion: an experimental study," Journal of Risk and Uncertainty, vol. 8, no. 3, pp. 289-307, 1994.

[34] J. Quiggin, "Regret theory with general choice sets," Journal of Risk and Uncertainty, vol. 8, no. 2, pp. 153-165, 1994.

[35] J. G. Wardrop, "Road paper. Some theoretical aspects of road traffic research," Proceedings of the Institution of Civil Engineers, vol. 1, no. 3, pp. 325-362, 1952.

[36] E. Avineri, "The effect of reference point on stochastic network equilibrium," Transportation Science, vol. 40, no. 4, pp. 409-420, 2006. 